\title{
GENERALIZATIONS OF KÄHLER-RICCI SOLITONS ON PROJECTIVE BUNDLES
}

\author{
GIDEON MASCHLER and CHRISTINA W. TØNNESEN-FRIEDMAN
}

\begin{abstract}
We prove that an admissible manifold (as defined by Apostolov, Calderbank, Gauduchon and Tønnesen-Friedman), arising from a base with a local Kähler product of constant scalar curvature metrics, admits Generalized Quasi-Einstein Kähler metrics (as defined by D. Guan) in all "sufficiently small" admissible Kähler classes. We give an example where the existence of Generalized Quasi-Einstein metrics fails in some Kähler classes while not in others. We also prove an analogous existence theorem for an additional metric type, defined by the requirement that the scalar curvature is an affine combination of a Killing potential and its Laplacian.
\end{abstract}

\section{Introduction}

In [6], [7], Guan defined and studied Generalized Quasi-Einstein (GQE) Kähler metrics. On compact manifolds, these are Kähler metrics for which the Ricci potential is also a Killing potential. This notion includes gradient Ricci solitons as a special case, and is thus a natural object of study (such solitons are called Quasi-Einstein metrics in some Physics references). In [7], GQE metrics are studied in relation to a modified Calabi flow. Finally, like extremal Kähler metrics, GQE metrics generalize the notion of constant scalar curvature (CSC) Kähler metrics.

Extremal Kähler metrics, defined by the requirement that the scalar curvature is a Killing potential, are the focus of much recent work in Kähler geometry. In [2], a continuity technique was used to show existence of certain explicit extremal metrics. Our aim in this paper is to apply the same technique to the question of existence of GQE metrics.

Existence of GQE metrics has been demonstrated in [6], [7], and [11] in all Kähler classes of certain manifolds. Here we consider a broader class of spaces, namely projective bundles over local products of CSC Kähler manifolds that are admissible in the sense defined in [2]. On these spaces we look for a particular type of GQE metric, which we call admissible. Our main results are as follows. First, we show that any admissible manifold admits a GQE metric in all admissible Kähler classes which are "small" in an appropriate sense. On

Received 1 September 2009, in final form 27 May 2010. 
the other hand, we give an example of a Kähler class on an admissible manifold which is not small, and contains no GQE metric.

Our work is laid out as follows. Section 2 provides a brief introduction to the Generalized Quasi-Einstein metrics as defined by D. Guan in [6] and [7]. Section 3 outlines a brief introduction to the notion of admissible manifolds, defined in [2], while Section 4 covers the definition and basic properties of admissible Generalized Quasi-Einstein metrics. Section 5 presents our existence theorem, achieved using a continuity argument. This is the heart and main purpose of these notes. Section 6 provides a non-existence example. Finally, Section 7 contains an appendix discussing another distinguished metric type of Guan, for which an analog of the main existence result is obtained.

We would like to thank Vestislav Apostolov for his helpful advice while preparing this paper. Some of the calculations in Sections 6 and 7 were carried out using the symbolic computation program Mathematica.

\section{Background}

Generalized Quasi-Einstein (GQE) Kähler metrics were first introduced by D. Guan [6]. They may be viewed as an alternative (with respect to extremal Kähler metrics) generalization of constant scalar curvature (CSC) Kähler metrics. For instance, any admissible geometrically ruled surface of genus higher than one has Kähler classes with no extremal metrics (but some Kähler classes on such a manifold do admit extremal metrics [12], [2], [13]). In [11] (see also [7] which offers a generalization) it is shown that any Kähler class on this type of manifold admits a GQE metric.

Let $M$ be a complex manifold with almost complex tensor $J$ and a Kähler metric $g$. A function $\phi$ on $M$ is called a Killing potential if $J \operatorname{grad} \phi$ is a Killing vector field (i.e., $\nabla J \operatorname{grad} \phi$ is skew-adjoint at every point).

Definition 2.1 ([6], [7]). Let $g$ be a Kähler metric on a compact complex manifold $(M, J)$, Scal its scalar curvature and $\overline{\text { Scal }}$ its average scalar curvature. We say that $g$ is a GQE metric if there exists a Killing potential $\phi$ for which

$$
\text { Scal }-\overline{\mathrm{Scal}}=\Delta \phi
$$

Here $\Delta$ denotes the Laplacian with respect to $g$.

Remark 2.2. Since $M$ is compact and Scal $-\overline{\mathrm{Scal}}=\Delta G$ Scal, with $G$ the Green operator, Definition 2.1 is equivalent to the requirement that the Ricci potential $-G$ Scal is also a Killing potential. In comparison, the definition of an extremal Kähler metric is equivalent to the statement that Scal itself is a Killing potential [3]. 
Definition 2.3 ([4], [5]). Let $\omega$ be a Kähler form on a compact complex manifold $(M, J)$ and let $h(M)$ denote the Lie algebra of the holomorphic vector fields on $(M, J)$. Then the Futaki invariant of $[\omega]$ is the map $\mathscr{F}_{[\omega]}: h(M) \rightarrow \mathrm{C}$ given by

$$
\mathscr{F}_{[\omega]}(\Xi)=-\int_{M} \Xi(G \mathrm{Scal}) d \mu,
$$

where $\Xi \in h(M)$ and $d \mu$ denotes the volume form of $\omega$.

The Futaki invariant is a Kähler class invariant. The class of any CSC Kähler metric has vanishing Futaki invariant. Moreover,

Proposition 2.4 ([6]). A GQE metric is CSC if and only if the Futaki invariant of the Kähler class vanishes.

Proof. We only need to check the "if" part of the statement. Suppose $g$ is a GQE metric as above for some Killing potential $\phi$. Then the value $\mathscr{F}_{[\omega]}\left((\bar{\partial} \phi)^{\sharp}\right)$ of the Futaki invariant on the holomorphic vector field $(\bar{\partial} \phi)^{\sharp}$ is equal to

$$
-\frac{1}{2} \int_{M}(\mathrm{Scal}-\overline{\mathrm{Scal}}) \phi d \mu=-\frac{1}{2} \int_{M} \phi \Delta \phi d \mu=-\frac{1}{2} \int_{M}\|d \phi\|^{2} d \mu,
$$

where the first equality follows from (1) (see e.g., [10]). If this expression vanishes, then $\phi$ is constant, and so, using (1) again, Scal $=\overline{\text { Scal }}$.

\section{Review of admissible manifolds and metrics}

Let $S$ be a compact complex manifold admitting a Kähler local product metric, whose components are Kähler metrics denoted $\left( \pm g_{a}, \pm \omega_{a}\right)$, and indexed by $a \in \mathscr{A} \subset \mathrm{Z}^{+}$. Here $\left( \pm g_{a}, \pm \omega_{a}\right)$ is the Kähler structure. In this notation we allow for the tensors $g_{a}$ to possibly be negatively definite - a parametrization given later justifies this convention. Note that in all our applications, each $\pm g_{a}$ is assumed to have CSC. The real dimension of each component is denoted $2 d_{a}$, while the scalar curvature of $\pm g_{a}$ is given as $\pm 2 d_{a} s_{a}$. Next, let $E_{0}, E_{\infty}$ be projectively flat hermitian holomorphic vector bundles over $S$, of ranks $d_{0}+1$ and $d_{\infty}+1$, respectively, such that the condition $c_{1}\left(E_{\infty}\right) /\left(d_{\infty}+1\right)-$ $c_{1}\left(E_{0}\right) /\left(d_{0}+1\right)=\sum_{a \in \mathscr{A}}\left[\omega_{a} / 2 \pi\right]$ holds. Then, following [2], the total space of the projectivization $M=P\left(E_{0} \oplus E_{\infty}\right) \rightarrow S$ is called admissible. A particular type of Kähler metric on $M$, also called admissible, will now be described.

Let $\hat{\mathscr{A}} \subset \mathrm{N} \cup \infty$ be the extended index set defined as follows:

- $\hat{A}=\mathscr{A}$, if $d_{0}=d_{\infty}=0$.

- $\hat{\mathscr{A}}=\mathscr{A} \cup\{0\}$, if $d_{0}>0$ and $d_{\infty}=0$.

- $\hat{A}=\mathscr{A} \cup\{\infty\}$, if $d_{0}=0$ and $d_{\infty}>0$.

- $\hat{A}=\mathscr{A} \cup\{0\} \cup\{\infty\}$, if $d_{0}>0$ and $d_{\infty}>0$. 
In the cases where $\hat{\mathscr{A}} \neq \mathscr{A}$, the following notations will prove useful: $x_{0}=1$, $x_{\infty}=-1, s_{0}=d_{0}+1$ and $s_{\infty}=-\left(d_{\infty}+1\right)$. Correspondingly, $\left(g_{0}, \omega_{0}\right)$ (or $\left.\left(g_{\infty}, \omega_{\infty}\right)\right)$ will be the induced Fubini-Study structure with scalar curvature $d_{0}\left(d_{0}+1\right)$ (or $\left.d_{\infty}\left(d_{\infty}+1\right)\right)$ on each fiber of $P\left(E_{0}\right)$ (or $\left.P\left(E_{\infty}\right)\right)$.

An admissible Kähler metric is constructed as follows. Consider the circle action on $M$ induced by the standard circle action on $E_{0}$. It extends to a holomorphic $\mathrm{C}^{*}$ action. The open and dense set $M_{0}$ of stable points with respect to the latter action has the structure of a principal circle bundle over the stable quotient. The hermitian norm on the fibers induces via a Legendre transform a function $z: M_{0} \rightarrow(-1,1)$ whose extension to $M$ consists of the critical manifolds $z^{-1}(1)=P\left(E_{0} \oplus 0\right)$ and $z^{-1}(-1)=P\left(0 \oplus E_{\infty}\right)$. Letting $\theta$ be a connection one form for the Hermitian metric on $M_{0}$, with curvature $d \theta=$ $\sum_{a \in \hat{\mathscr{A}}} \omega_{a}$, an admissible Kähler metric and form are given up to scale by the respective formulas

(2) $g=\sum_{a \in \hat{\mathscr{A}}} \frac{1+x_{a} z}{x_{a}} g_{a}+\frac{d z^{2}}{\Theta(z)}+\Theta(z) \theta^{2}, \quad \omega=\sum_{a \in \hat{\mathscr{A}}} \frac{1+x_{a} z}{x_{a}} \omega_{a}+d z \wedge \theta$,

valid on $M_{0}$. Here $\Theta$ is a smooth function with domain containing $(-1,1)$ and $x_{a}, a \in \mathscr{A}$ are real numbers of the same sign as $g_{a}$ and satisfying $0<\left|x_{a}\right|<1$. The complex structure yielding this Kähler structure is given by the pullback of the base complex structure along with the requirement $J d z=\Theta \theta$. The function $z$ is hamiltonian with $K=J \operatorname{grad} z$ a Killing vector field. In fact, $z$ is the moment map on $M$ for the circle action, decomposing $M$ into the free orbits $M_{0}=z^{-1}((-1,1))$ and the special orbits $z^{-1}( \pm 1)$. Finally, $\theta$ satisfies $\theta(K)=1$.

In order that $g$ (be a genuine metric and) extend to all of $M, \Theta$ must satisfy the positivity and boundary conditions

(i) $\Theta(z)>0, \quad-1<z<1$,

(ii) $\Theta( \pm 1)=0$,

(iii) $\Theta^{\prime}( \pm 1)=\mp 2$.

The last two of these are together necessary and sufficient for the compactification of $g$.

The Kähler class $\Omega_{x}=[\omega]$ of an admissible metric is also called admissible and is uniquely determined by the parameters $x_{a}, a \in \mathscr{A}$, once the data associated with $M$ (i.e., $d_{a}, s_{a}, g_{a}$ etc.) is fixed. The $x_{a}, a \in \mathscr{A}$, together with the data associated with $M$ will be called admissible data. The reader is urged to consult Section 1 of [2] for further background on this set-up. 
Define a function $F(z)$ by the formula $\Theta(z)=F(z) / p_{\mathrm{c}}(z)$, where $p_{\mathrm{c}}(z)=$ $\prod_{a \in \hat{A}}\left(1+x_{a} z\right)^{d_{a}}$. Since $p_{\mathrm{c}}(z)$ is positive for $-1<z<1$, conditions (3) imply the following conditions on $F(z)$, which are only necessary for compactification of the metric $g$ :

$$
\begin{aligned}
& \text { (i) } F(z)>0, \quad-1<z<1 \text {, } \\
& \text { (ii) } F( \pm 1)=0, \\
& \text { (iii) } F^{\prime}( \pm 1)=\mp 2 p_{c}( \pm 1) .
\end{aligned}
$$

For the purpose of understanding admissible GQE metrics, it is useful to recall the fact below.

Proposition 3.1 ([1]). For any admissible metric $g$, if $S(z)$ is a smooth function of $z$, then

$$
\Delta S=-\left[F(z) S^{\prime}(z)\right]^{\prime} / p_{c}(z)
$$

where $\Delta$ is the Laplacian of $g$.

Proof. This is a special case of Lemma 5 in [1], but for convenience we shall review the proof here. We denote by $(.,-$ ) the inner product on two forms induced by $g$. Recall that

$$
\Delta S=-\left(d d^{c} S(z), \omega\right)=-(d J d S(z), \omega) .
$$

Thus

$$
\begin{aligned}
-\Delta S= & \left(d\left(S^{\prime}(z) J d z\right), \omega\right)=\left(d\left(S^{\prime}(z) \frac{F(z)}{p_{c}(z)} \theta\right), \omega\right) \\
= & \left(\left(\frac{\left[S^{\prime}(z) F(z)\right]^{\prime}}{p_{c}(z)}-\frac{S^{\prime}(z) F(z) p_{c}^{\prime}(z)}{\left(p_{c}(z)\right)^{2}}\right) d z \wedge \theta, \omega\right) \\
& \quad+\left(S^{\prime}(z) \frac{F(z)}{p_{c}(z)} \sum_{a \in \hat{A}} \omega_{a}, \omega\right) \\
= & \frac{\left[S^{\prime}(z) F(z)\right]^{\prime}}{p_{c}(z)}-\frac{S^{\prime}(z) F(z)}{p_{c}(z)}\left[\frac{p_{c}^{\prime}(z)}{\left(p_{c}(z)\right)}-\sum_{a \in \hat{\mathscr{A}}} \frac{d_{a} x_{a}}{\left(1+x_{a} z\right)}\right] \\
= & \frac{\left[S^{\prime}(z) F(z)\right]^{\prime}}{p_{c}(z)},
\end{aligned}
$$

where the relation $\left(\omega_{a}, \omega\right)=\left(\omega_{a},\left(\left(1+x_{a} z\right) / x_{a}\right) \omega_{a}\right)=\left(x_{a} /\left(1+x_{a} z\right)\right)^{2}\left(\omega_{a}\right.$, $\left.\left(\left(1+x_{a} z\right) / x_{a}\right) \omega_{a}\right)_{a}=\left(x_{a} /\left(1+x_{a} z\right)\right) d_{a}$, with $\left({ }_{-},{ }_{-}\right)_{a}$ the inner product induced by $g_{a}$, was used. 
The scalar curvature of an admissible metric is given (cf. [1], or (10) in [2]) by

$$
\mathrm{Scal}=\sum_{a \in \hat{\mathscr{A}}} \frac{2 d_{a} s_{a} x_{a}}{1+x_{a} z}-\frac{F^{\prime \prime}(z)}{p_{\mathrm{c}}(z)},
$$

Let $C_{*}^{\infty}([-1,1])$ denote the set of functions $f(z)$ of $z$ which are smooth in $[-1,1]$ and normalized so that they integrate to zero when viewed as smooth functions on $M$. The latter condition is equivalent to $\int_{-1}^{1} f(z) p_{c}(z) d z=0$, since the volume form of an admissible metric equals $p_{c}(z)\left(\bigwedge_{a} \frac{\left(\omega_{a} / x_{a}\right)^{d_{a}}}{d_{a} !}\right) \wedge$ $d z \wedge \theta$.

COROLlary 3.2. Given an admissible metric g, its Laplacian gives a surjective map from $C_{*}^{\infty}([-1,1])$ to itself (considered as a space of functions on $M)$.

Proof. Given $R(z) \in C_{*}^{\infty}([-1,1])$, an explicit solution to $\Delta S(z)=R(z)$ can be obtained directly from (5) on the open dense set for which $z \neq \pm 1$. Either by Hodge decomposition for smooth functions on compact manifolds or, more concretely, by a L'hospital rule argument (using (4.ii) and (4.iii)), this solution extends to the \pm 1 level sets of $z$.

COROLlary 3.3. The Ricci potential of an admissible metric is a function of $z$.

Proof. This follows from the previous corollary since by (6) the scalar curvature of an admissible metric is a smooth function of the moment map $z$.

\section{GQE metrics on admissible manifolds}

Recall from Remark 2.2 that a Kähler metric is GQE if and only if its Ricci potential is a Killing potential. It follows from Corollary 3.3 that an admissible metric $g$ with moment map $z$ is GQE only if its Ricci potential is affine in $z$. When this holds, we will call the metric admissible GQE. Using Definition 2.1 and Remark 2.2, the admissible GQE condition can be written as

$$
\text { Scal }-\overline{\text { Scal }}=k \Delta z
$$

for some $k \in \mathrm{R}$.

We turn now to an ODE for $F$ which characterizes admissible GQE metrics. Since for an admissible metric we have from (6) and (5) the formulas

$$
\mathrm{Scal}=\sum_{a \in \hat{\mathscr{A}}} \frac{2 d_{a} s_{a} x_{a}}{1+x_{a} z}-\frac{F^{\prime \prime}(z)}{p_{\mathrm{c}}(z)}, \quad \Delta z=\frac{-F^{\prime}(z)}{p_{\mathrm{c}}(z)},
$$


equation (7) holds if and only if

$$
F^{\prime \prime}(z)-k F^{\prime}(z)=2\left(\sum_{a \in \hat{A}} \frac{d_{a} s_{a} x_{a}}{1+x_{a} z}\right) p_{\mathrm{c}}(z)-\frac{2 \beta_{0} p_{\mathrm{c}}(z)}{\alpha_{0}},
$$

where

$$
\alpha_{0}=\int_{-1}^{1} p_{\mathrm{c}}(t) d t
$$

and

$$
\beta_{0}=p_{\mathrm{c}}(1)+p_{\mathrm{c}}(-1)+\int_{-1}^{1}\left(\sum_{a \in \hat{A}} \frac{d_{a} s_{a} x_{a}}{1+x_{a} t}\right) p_{\mathrm{c}}(t) d t .
$$

Note here that the expression $\overline{\mathrm{Scal}}=2 \beta_{0} / \alpha_{0}$ (as well as the formula for Scal), appears in the proof of Proposition 6 in [2].

REMARK 4.1. Using work of [2], it is straightforward to verify that an admissible metric is simultaneously GQE and extremal if and only if it is CSC. It is tempting to conjecture that this is true in more generality.

Just as in the extremal case (see e.g., Section 2.4 in [2]), equations (4.ii) and (4.iii) together with (8) imply (3.ii) and (3.iii). So, under assumption (8), (4.ii) and (4.iii) are the necessary and sufficient boundary conditions for the compactification of $g$.

Integrating (8) and then solving the resulting first order ODE gives

$$
F(z)=e^{k z} \int_{-1}^{z} e^{-k t} P(t) d t
$$

where $k$ is a constant and

$$
P(t)=2 \int_{-1}^{t}\left(\left(\sum_{a \in \hat{A}} \frac{d_{a} s_{a} x_{a}}{1+x_{a} u}\right) p_{\mathrm{c}}(u)-\frac{\beta_{0} p_{\mathrm{c}}(u)}{\alpha_{0}}\right) d u+2 p_{\mathrm{c}}(-1),
$$

with the last term determined by the requirement that (4.iii) be satisfied. Also, (4.ii) will be satisfied if and only if there exists a $k \in \mathrm{R}$ for which

$$
\int_{-1}^{1} e^{-k t} P(t) d t=0
$$

In summary, we have

Proposition 4.2. Given admissible data on an admissible manifold, let $F$ be the solution of (8) of the form (9), (10). Suppose there exists $k \in \mathbf{R}$ for which (11) holds and (4.i) is satisfied by F. Then the admissible metric 
naturally constructed from $F$ and the given data is GQE. Conversely, for any admissible GQE metric (up to scale), the associated function $F$ has the form (9), (10), solves (8), satisfies (4.i) and there exists a $k \in \mathrm{R}$ for which (11) holds.

We give now two preparatory lemmas on properties of the rational function $P(t)$.

LEMma 4.3. For any given admissible data, the function $P(t)$ given by (10) satisfies: If $d_{0}=0$, then $P(-1)>0$, otherwise $P(-1)=0$. If $d_{\infty}=0$, then $P(1)<0$, otherwise $P(1)=0$. Furthermore, $P(t)>0$ in some (deleted) right neighborhood of $t=-1$, and $P(t)<0$ in some (deleted) left neighborhood of $t=1$.

Proof. First observe that by design $P( \pm 1)=\mp 2 p_{\mathrm{c}}( \pm 1)$, which yields the claimed signs of $P$ at the endpoints. Also, $p_{\mathrm{c}}(t)$ contains the factors $1+x_{0} t$, $1+x_{\infty} t$ with multiplicity $d_{0}$ or, respectively, $d_{\infty}$. One of these factors accounts for the vanishing of $P$ at $t=-1$ (or $t=1$ ) unless $d_{0}=0$ (or $d_{\infty}=0$ ). Furthermore, $P^{\prime}(t)$ contains these factors in each summand, to multiplicity at least $d_{0}-1$ (or $d_{\infty}-1$ ). Differentiating $P(t)$, we see that if $d_{0}>0$, then $P^{\left(d_{0}\right)}(-1)>0$ (and the lower order derivatives vanish), while if $d_{\infty}>0$, then $P^{\left(d_{\infty}\right)}(1)$ has sign $(-1)^{d_{\infty}+1}$ (and the lower order derivatives vanish). From these observations the result follows easily by considering the Taylor expansion of $P(t)$ near \pm 1 .

Lemma 4.4. If the function $P(t)$ given by (10) has exactly one root in the interval $(-1,1)$, then there exists a unique $k \in \mathrm{R}$ such that

$$
\int_{-1}^{1} e^{-k t} P(t) d t=0
$$

Moreover, for this $k$, the positivity condition (4.i) is satisfied when $F(z)$ is defined as in (9), (10).

Proof. If $P(t)$ has just one root $t_{0}$ in the interval $(-1,1)$, then, we may write

$$
P(t)=\left(t-t_{0}\right) p(t)
$$

where, due to Lemma 4.3, $p(t)$ is negative for all $t \in(-1,1)$. Consider now the auxiliary function

$$
G(k)=e^{k t_{0}} \int_{-1}^{1} e^{-k t} P(t) d t=\int_{-1}^{1} p(t)\left(t-t_{0}\right) e^{-k\left(t-t_{0}\right)} d t .
$$

By direct calculation, $G^{\prime}(k)$ is positive, while $\lim _{k \rightarrow-\infty} G=-\infty$, and $\lim _{k \rightarrow \infty} G=+\infty$, as can be checked by taking the limit after first breaking 
the integral in the form $\int_{-1}^{t_{0}}+\int_{t_{0}}^{1}$. This proves the existence and uniqueness of a $k$ for which $G(k)=0$, or equivalently $\int_{-1}^{1} e^{-k t} P(t) d t=0$.

Finally, given this $k$, since $e^{-k t} P(t)$ changes sign exactly once in $(-1,1)$ and is positive near $t=-1$, condition (12) clearly guarantees that $\int_{-1}^{z} e^{-k t} P(t) d t$ is a nonnegative function for $z \in(-1,1)$. Therefore (4.i) is satisfied for $F(z)$ as defined in (9), (10).

\section{A continuity argument}

Let $M=P\left(E_{0} \oplus E_{\infty}\right) \rightarrow S$ be an admissible manifold, where the base $S$ is a local Kähler product of CSC metrics $\left( \pm g_{a}, \pm \omega_{a}\right)$. The aim of this section is to show that for $\left|x_{a}\right|$ sufficiently small for all $a \in \mathscr{A}$, the corresponding Kähler class admits an admissible GQE metric. In light of Lemma 4.4, the strategy will be to show that in this case $P(t)$ has just one root in $(-1,1)$.

Observe that

$$
P^{\prime}(t)=2\left(\sum_{a \in \hat{\mathscr{A}}} \frac{d_{a} s_{a} x_{a}}{1+x_{a} t}\right) p_{\mathrm{c}}(t)-\frac{2 \beta_{0} p_{\mathrm{c}}(t)}{\alpha_{0}}
$$

and, as in the proof of Lemma 4.3, we make the following observations

- If $d_{0}>1$, then $P^{\prime}(-1)=0$ and $P^{\prime}(t)$ is positive in some (deleted) right neighborhood of $t=-1$.

- If $d_{0}=1$, then $P^{\prime}(-1)>0$.

- If $d_{\infty}>1$, then $P^{\prime}(1)=0$ and $P^{\prime}(t)$ is positive in some (deleted) left neighborhood of $t=-1$.

- If $d_{\infty}=1$, then $P^{\prime}(1)>0$.

We will now look at the behaviour of $P^{\prime}(t)$ when $x_{a}$ is near 0 for all $a \in \mathscr{A}$. The limit $x_{a} \rightarrow 0$ for all $a \in \mathscr{A}$ (of any expression) will be denoted simply by lim. This limit cannot be taken in the formulae for admissible Kähler metric and class, but $P(t)$ defined in (10) and $P^{\prime}(t)$ above, with $x_{a}=0$, are still well-defined functions.

Lemma 5.1. $\lim P^{\prime}(t)$, taken as $x_{a} \rightarrow 0$ for all $a \in \mathscr{A}$, equals

$$
\begin{gathered}
2 d_{0}\left(d_{0}+1\right)(1+t)^{d_{0}-1}(1-t)^{d_{\infty}} \\
+2 d_{\infty}\left(d_{\infty}+1\right)\left((1+t)^{d_{0}}(1-t)^{d_{\infty}-1}\right. \\
-\left(1+d_{0}+d_{\infty}\right)\left(2+d_{0}+d_{\infty}\right)(1+t)^{d_{0}}(1-t)^{d_{\infty}} .
\end{gathered}
$$

Proof. The first two terms of the expression simply follows from the fact that $s_{0} x_{0}=d_{0}+1$ (if $d_{0} \neq 0$ ) and $s_{\infty} x_{\infty}=d_{\infty}+1$ (if $d_{\infty} \neq 0$ ). 
The last term follows from the fact that (in the limit considered here) $\lim \left(2 \beta_{0} / \alpha_{0}\right)$ equals $\left(1+d_{0}+d_{\infty}\right)\left(2+d_{0}+d_{\infty}\right)$. This fact is not at all trivial but follows directly from the calculations at the end of Appendix B of [2].

The following cases occur for $\lim P^{\prime}(t)$.

\subsection{Case 1: $d_{0}>0, d_{\infty}>0$}

In this case $\lim P^{\prime}(t)$ is

$$
g(t)(1+t)^{d_{0}-1}(1-t)^{d_{\infty}-1}
$$

where

$$
\begin{aligned}
g(t)=2 d_{0}\left(d_{0}+1\right)(1-t)+2 & d_{\infty}\left(d_{\infty}+1\right)(1+t) \\
& -\left(1+d_{0}+d_{\infty}\right)\left(2+d_{0}+d_{\infty}\right)\left(1-t^{2}\right)
\end{aligned}
$$

is a concave up parabola, which is positive at $t= \pm 1$ and has a minimum value equal to $-4\left(1+d_{0}\right)\left(1+d_{\infty}\right) /\left(2+d_{0}+d_{\infty}\right)$, so negative, in the interval $(-1,1)$. It is now clear that $\lim P^{\prime}(t)$ has two distinct simple roots in the interval $(-1,1)$. Thus for $\left|x_{a}\right|$ sufficiently small for all $a \in \mathscr{A}$, the function $P^{\prime}(t)$ also has exactly two zeroes, i.e., $P(t)$ has exactly two critical points in $(-1,1)$. This is because the factored term $(1+t)^{d_{0}-1}(1-t)^{d_{\infty}-1}$ does not depend on $x_{a}$, so the corresponding endpoint roots stay put as $x_{a}$ changes. Putting this together with Lemma 4.3, we see that $P(t)$ must change sign exactly once in $(-1,1)$.

\subsection{Case 2: $d_{0}=0, d_{\infty}>0$}

In this case $\lim P^{\prime}(t)$ is

$$
g(t)\left(1+d_{\infty}\right)(1-t)^{d_{\infty}-1}
$$

where

$$
g(t)=\left(2+d_{\infty}\right) t+d_{\infty}-2
$$

is linear and increasing from $g(-1)=-4<0$ to $g(1)=2 d_{\infty}>0$. Hence $\lim P^{\prime}(t)$ has exactly one simple zero in $(-1,1)$. Thus for $\left|x_{a}\right|$ sufficiently small for all $a \in \mathscr{A}$, the function $P^{\prime}(t)$ also has exactly one zero, i.e., $P(t)$ has exactly one critical point in $(-1,1)$. Putting this together with Lemma 4.3, we see that $P(t)$ must change sign exactly once in $(-1,1)$. 
5.3. Case 3: $d_{0}>0, d_{\infty}=0$

In this case $\lim P^{\prime}(t)$ is

$$
g(t)\left(1+d_{0}\right)(1+t)^{d_{0}-1},
$$

where

$$
g(t)=-\left(2+d_{0}\right) t+d_{0}-2
$$

is linear and decreasing from $g(-1)=2 d_{0}>0$ to $g(1)=-4>0$. Hence $\lim P^{\prime}(t)$ has exactly one simple root in $(-1,1)$. Thus for $\left|x_{a}\right|$ sufficiently small for all $a \in \mathscr{A}$, the function $P^{\prime}(t)$ also has exactly one zero, i.e., $P(t)$ has exactly one critical point in $(-1,1)$. Putting this together with Lemma 4.3 , we see that $P(t)$ must change sign exactly once in $(-1,1)$.

5.4. Case 4: $d_{0}=0=d_{\infty}$

In this case $\lim P^{\prime}(t)$ is simply the constant function $g(t)=-2$. Hence $\lim P^{\prime}(t)$ has no roots in $(-1,1)$ and is negative. Thus for $\left|x_{a}\right|$ sufficiently small for all $a \in \mathscr{A}$, the function $P^{\prime}(t)$ is also strictly negative, i.e., $P(t)$ is a strictly decreasing function on $(-1,1)$. Putting this together with Lemma 4.3, we see that $P(t)$ must change sign exactly once in $(-1,1)$.

Having thus considered all possible cases we may now conclude with

Theorem 5.2. Let $M=P\left(E_{0} \oplus E_{\infty}\right) \rightarrow S$ be an admissible manifold arising from a base $S$ with a local Kähler product of CSC metrics. Then the set of admissible Kähler classes admitting an admissible GQE metric forms a nonempty open subset of the set of all admissible Kähler classes. Any admissible Kähler class which is sufficiently small, that is, for which $\left|x_{a}\right|, a \in \mathscr{A}$, are all sufficiently small, belongs to this subset.

Proof. The non-emptiness and the inclusion of sufficently small admissible classes follow from the observations above and Lemma 4.4.

For the openness we proceed as follows. Recall from Section 3 that for a given admissible manifold, the admissible Kähler classes are parameterized (up to scale) by $x_{a}, a \in \mathscr{A}$. Suppose $\mathscr{A}=\{1, \ldots, N\}$, so that the set of admissible Kähler classes (up to scale) is represented by an open subset $W \subset(-1,1)^{N}$. Rephrasing Proposition 4.2, an admissible Kähler class given by $\left(x_{1}, \ldots, x_{N}\right)$ admits an admissible GQE metric if and only if there exists $k \in \mathrm{R}$ such that

and

$$
\int_{-1}^{1} e^{-k t} P(t) d t=0
$$

for $P(t)$ as in (10). 
Suppose that $\left(x_{1}^{0}, \ldots, x_{N}^{0}, k^{0}\right) \in W \times \mathrm{R}$ satisfies (13) and (14). We need to show that for $\left(x_{1}, \ldots, x_{N}\right) \in W$ sufficiently close to $\left(x_{1}^{0}, \ldots, x_{N}^{0}\right)$, there exists $k \in \mathrm{R}$ such that $\left(x_{1}, \ldots, x_{N}, k\right)$ also satisfies (13) and (14). Define $\Phi: W \times \mathrm{R} \rightarrow \mathrm{R}$ by

$$
\Phi\left(x_{1}, \ldots, x_{N}, k\right)=\int_{-1}^{1} e^{-k t} P(t) d t,
$$

where $P(t)$ is determined by $\left(x_{1}, \ldots, x_{N}\right)$. Clearly $\Phi$ is a smooth mapping. Then

$$
\begin{aligned}
\frac{\partial \Phi}{\partial k} & =-\int_{-1}^{1} t e^{-k t} P(t) d t \\
& =-\int_{-1}^{1} e^{-k t} P(t) d t+\int_{-1}^{1}\left(\int_{-1}^{t} e^{-k u} P(u) d u\right) d t,
\end{aligned}
$$

which by (13) and (14) is positive at $\left(x_{1}^{0}, \ldots, x_{N}^{0}, k^{0}\right)$. A standard implicit function theorem now gives an open neighborhood $U \subset W$ of $\left(x_{1}^{0}, \ldots, x_{N}^{0}\right)$ such that for all $\left(x_{1}, \ldots, x_{N}\right) \in U$ there exists $k \in \mathrm{R}$ such that $\Phi\left(x_{1}, \ldots, x_{N}, k\right)=$ 0 , i.e., (13) is satisfied. Moreover, such $k$ are close to $k^{0}$, when $\left(x_{1}, \ldots, x_{N}\right)$ is close to $\left(x_{1}^{0}, \ldots, x_{N}^{0}\right)$. By continuity of $\int_{-1}^{t} e^{-k u} P(u) d u$ with respect to $x_{1}, \ldots, x_{N}$, and $k$, there is an open neighborhood $V \subset U \subset W$ of $\left(x_{1}^{0}, \ldots, x_{N}^{0}\right)$ such that for each $\left(x_{1}, \ldots, x_{N}\right) \in V$ there exists $k \in \mathrm{R}$ for which (14) as well as (13) are satisfied. The openness statement now follows, and this concludes the proof of Theorem 5.2.

An alternative way to obtain a setting where the assumption in Lemma 4.4 is met, is to put conditions on the sign distribution of $s_{a} x_{a}$ and then perform a delicate root counting argument on $P^{\prime}(t)$ similar in type to the one encountered for extremal Kähler metrics (see Hwang and Singer [9] as well as Guan [8]). This is exactly D. Guan's method in [7]. For certain types of admissible manifolds his method yields existence of GQE metrics thoughout the Kähler cone. For most cases though, the existence of GQE metrics obtained in this way will depend on the choice of Kähler class - in a different sense than our "smallness" condition - as well as on the particular admissible manifold.

\section{A non-existence example}

Consider the admissible manifold

$$
P(\mathscr{O} \oplus \mathscr{O}(1,-1)) \rightarrow \Sigma_{1} \times \Sigma_{2},
$$

where $\Sigma_{1}$ and $\Sigma_{2}$ are both compact Riemann surfaces of genus two and $g_{1}$ and $-g_{2}$ are both Kähler metrics of scalar curvature -4 . Thus $d_{0}=d_{\infty}=0$, 
$\hat{\mathscr{A}}=\mathscr{A}=\{1,2\}, d_{1}=d_{2}=1, s_{1}=-s_{2}=-2$, and the Kähler cone is parametrized by $0<x_{1}<1$ and $-1<x_{2}<0$.

Using Proposition 6 in [2] one may calculate that the Futaki invariant of $J \operatorname{grad} z$ equals (up to sign and scale)

$$
\frac{\left(1+x_{1}-x_{2}\right)\left(x_{1}+x_{2}\right)}{\left(3+x_{1} x_{2}\right)^{2}} .
$$

When $x_{2}=-x_{1}$ this vanishes, in fact $\mathscr{F}_{[\omega]}(\Xi)$ vanishes for any $\Xi \in$ $h(M) \cong \mathrm{C}^{\times}$, and using Proposition 2.4 we see that any GQE metric in the corresponding class must be CSC. In turn, any CSC Kähler metric must be admissible [2], and thus $k$ in equation (7) should equal zero. Calculating $P(t)$ in this case, we get

$$
P(t)=\frac{2 t\left(3-3 x_{1}^{2}-4 x_{1}^{3}-x_{1}^{2}\left(1-4 x_{1}-x_{1}^{2}\right) t^{2}\right)}{x_{1}^{2}-3} .
$$

It is easy to see that $\int_{-1}^{1} P(t) d t=0$, so $F(z)=\int_{-1}^{z} P(t) d t$ solves (4.ii) as well as (8) and (4.iii). We calculate that

$$
F(z)=\frac{\left(1-z^{2}\right)\left(6-7 x_{1}^{2}-4 x_{1}^{3}+x_{1}^{4}-x_{1}^{2}\left(1-4 x_{1}-x_{1}^{2}\right) z^{2}\right)}{2\left(3-x_{1}^{2}\right)} .
$$

For the interested reader, let us remark that $F(z)$ is the extremal polynomial introduced in [2].

By direct inspection (or by Theorem 2 in [2] and Theorem 5.2 in this text), we see that if $\left|x_{1}\right|$ is sufficiently small, (4.i) holds and a CSC metric exists in the corresponding Kähler class. However, for e.g., $x_{1}=0.8$ (and $x_{2}=-0.8$ ) (4.i) fails, and thus there is exists no GQE metric in the corresponding Kähler class.

Notice, that off but near the line $x_{2}=-x_{1}$, (e.g., $x_{1}=0.9$ and $\left.x_{2}=-0.75\right)$ one may check that there is no extremal Kähler metric in the corresponding class. It can, however, be shown that in this case $P(t)$ satisfies Lemma 4.4. Hence this Kähler class admits an admissible GQE metric.

Remark 6.1. It seems to be "easier" to obtain existence of an admissible GQE metric as compared to that of an (admissible) extremal Kähler metric in a given admissible Kähler class. It is tempting to conjecture that the existence of extremal Kähler metrics in admissible Kähler classes (i.e., positivity of the extremal polynomial) implies the existence of an admissible GQE metric. Such a result would yield Theorem 5.2 as a corollary of Theorem 2 from [2]. To determine this one would have to study more closely the relationship between the extremal polynomial from [2] and $P(z)$. 


\section{Appendix: other metrics}

The methods of this paper can be used to give an existence result for another distinguished metric type, which interpolates between extremal and GQE metrics. This type has been considered by Guan in [7]. Namely, the Killing potential $\phi$ is now required to satisfy an equation stating that $\mathrm{Scal}-\overline{\text { Scal }}$ is an affine combination of $\Delta \phi$ and $\phi$. Among admissible metrics with an associated moment map $z$, we therefore look for metrics satisfying

$$
\mathrm{Scal}-\overline{\mathrm{Scal}}=k \Delta z+b(z+l),
$$

for some $k, b, l \in \mathrm{R}$. The constant $l$ guarantees that the right hand side of this equation integrates to zero. It can be computed from admissible data using its defining equation (15), along with the expressions appearing in the proof of Proposition 6 of [2], giving $l=-\alpha_{1} / \alpha_{0}$, with $\alpha_{r}=\int_{-1}^{1} p_{c}(t) t^{r} d t, r=1,2$. Using Appendix B of [2], we have

Lemma 7.1. The limit of $l$ as $x_{a} \rightarrow 0$ for all $a \in \mathscr{A}$ is $\left(d_{\infty}-d_{0}\right) /(2+$ $\left.d_{0}+d_{\infty}\right)$.

We now state an existence result for metrics satisfying (15).

Theorem 7.2. Let $M=P\left(E_{0} \oplus E_{\infty}\right) \rightarrow S$ be an admissible manifold arising from a base $S$ with a local Kähler product of CSC metrics. Then, for any given $b \in \mathrm{R}$, the set of admissible Kähler classes admitting an admissible metric satisfying (15) forms a nonempty open subset in the set of all admissible Kähler classes. Any admissible Kähler class which is sufficiently small, that is, for which $\left|x_{a}\right|, a \in \mathscr{A}$, are all sufficiently small, belongs to this subset.

Remark 7.3. Aside from generalizing Theorem 5.2, the above theorem overlaps with Proposition 9 in [2], which says that for small classes we may solve (15) for $k=0$, obtaining an extremal Kähler metric. Moreover, a solution with $k=0$ can only exist with a particular - Kähler class dependent - value of $b$ (namely $-A$ as defined in Proposition 6 of [2], see also equation (13) there). Therefore, when $b$ does not equal this value and is not zero, Theorem 7.2 guarantees existence of Kähler metrics which are of a new type, i.e., are neither extremal nor GQE.

Below we only prove Theorem 7.2 in the case when the ranks of $E_{0}$ and $E_{\infty}$ are at least 2, i.e., when $d_{0}, d_{\infty}>0$. The general argument is similar.

PROOF. The ODE corresponding to (8) in this case, is

$$
F^{\prime \prime}(z)-k F^{\prime}(z)=2\left(\sum_{a \in \hat{\mathscr{A}}} \frac{d_{a} s_{a} x_{a}}{1+x_{a} z}\right) p_{\mathrm{c}}(z)-\left(\frac{2 \beta_{0}}{\alpha_{0}}+b(z+l)\right) p_{\mathrm{c}}(z),
$$


and again, assuming this equation holds, (4.ii) and (4.iii) are the necessary and sufficient boundary conditions, which guarantee existence of a metric of type (15) on a (compact) admissible manifold. Its solution $F$ satisfies, as before, $F(z)=e^{k z} \int_{-1}^{z} e^{-k t} P(t) d t$, where $P(t)$ (given similarly to (10)) is such that

$$
P^{\prime}(t)=2\left(\sum_{a \in \hat{A}} \frac{d_{a} s_{a} x_{a}}{1+x_{a} t}\right) p_{\mathrm{c}}(t)-\left(\frac{2 \beta_{0}}{\alpha_{0}}+b(t+l)\right) p_{\mathrm{c}}(t) .
$$

For the function $P(t)$, the analog of Lemma 4.3 holds (since the proof depends largely on $\left.p_{\mathrm{c}}(t)\right)$. The analog of Lemma 4.4 also holds, for fixed $b$ and $l$, with the same proof. Hence what is left is to analyze $\lim P^{\prime}(t)$, taken as $x_{a} \rightarrow 0$ for all $a \in \mathscr{A}$. As in Case 1, we have $\lim P^{\prime}(t)=g(t)(1+t)^{d_{0}-1}(1-t)^{d_{\infty}-1}$, yet here $g(t)$ is the cubic polynomial

$$
\begin{aligned}
g(t)= & 2 d_{0}\left(d_{0}+1\right)(1-t)+2 d_{\infty}\left(d_{\infty}+1\right)(1+t) \\
& -\left(1+d_{0}+d_{\infty}\right)\left(2+d_{0}+d_{\infty}\right)\left(1-t^{2}\right)-b(t+\lim l)\left(1-t^{2}\right) .
\end{aligned}
$$

We have $g(-1)=4 d_{0}\left(d_{0}+1\right)>0, g(1)=4 d_{\infty}\left(d_{\infty}+1\right)>0$. Hence (asymptotics of a cubic show that) one of the roots of $g(t)$ lies outside $(-1,1)$, and thus at most two lie in $(-1,1)$. Our proof will be complete once we show that $g(t)$ has exactly two simple roots in $(-1,1)$, since then the same will hold for $\lim P(t)$, and we can proceed as in the proof of Theorem 5.2. For this, it is enough to show that $g\left(t_{0}\right)<0$ for some $t_{0} \in(-1,1)$. Let $t_{0}=-\lim l=\left(d_{0}-d_{\infty}\right) /\left(2+d_{0}+d_{\infty}\right)$. This number clearly lies in $(-1,1)$, and a direct calculation gives $g\left(t_{0}\right)=-\left(4\left(1+d_{\infty}\right)\left(1+d_{0}\right)\right) /\left(2+d_{0}+d_{\infty}\right)<0$ as required. This completes the proof of non-emptiness and the inclusion of sufficiently small admissible classes, using Lemma 4.4. Openness follows as in Theorem 5.2.

Remark 7.4. It is not hard to check that the Kähler class in the example from Section 6, which carries no GQE nor extremal Kähler metric, does in fact have admissible metrics satisfying (15).

\section{REFERENCES}

1. Apostolov, V., Calderbank, D. M. J., and Gauduchon, P., Hamiltonian 2-forms in Kähler geometry I. General theory, J. Differential Geom. 73 (2006), 359-412.

2. Apostolov, V., Calderbank, D. M. J., Gauduchon, P., and C. Tønnesen-Friedman, Hamiltonian 2-forms in Kähler geometry III. Extremal metrics and stability, Invent. math. 173 (2008) 547-601.

3. Calabi, E., Extremal Kähler metrics, pp. 259-290 in: Seminar on Differential Geometry, Ann. Math. Stud. 102, Princeton Univ. Press, Princeton 1982. 
4. Calabi, E., Extremal Kähler metrics II, pp. 95-114 in: I. Chavel, H. M. Farkas (eds.), Differential Geometry and Complex Analysis, Springer, Berlin 1985.

5. Futaki, A., Kähler-Einstein Metrics and Integral Invariants, Lecture Notes in Math. 1314, Springer, Berlin 1988.

6. Guan, D., Quasi-Einstein metrics, Internat. J. Math. 6 (1995), 371-379.

7. Guan, D., Extremal solitons and exponential $C^{\infty}$ convergence of the modified Calabi flow on certain $\mathrm{CP} P^{1}$ bundles, Pacific J. Math. 233 (2007), 91-124.

8. Guan, D., Existence of extremal metrics on compact almost homogeneous Kähler manifolds with two ends, Trans. Amer. Math. Soc. 347 (1995), 2255-2262.

9. Hwang, A. D., and Singer, M. A., A momentum construction for circle-invariant Kähler metrics, Trans. Amer. Math. Soc. 354 (2002), 2285-2325.

10. LeBrun, C., and Simanca, S. R., Extremal Kähler metrics and complex deformation theory, Geom. Funct. Anal. 4 (1994), 298-335.

11. Pedersen, H., Tønnesen-Friedman, C., and Valent, G., Quasi-Einstein Kähler metrics, Lett. Math. Phys. 50 (1999), 229-241.

12. Székelyhidi, G., Extremal metrics and K-stability, Bull. Lond. Math. Soc. 39 (2007), 76-84.

13. Tønnesen-Friedman, C., Extremal Kähler metrics on minimal ruled surfaces, J. Reine Angew. Math. 502 (1998), 175-197.

DEPARTMENT OF MATHEMATICS AND COMPUTER SCIENCE

CLARK UNIVERSITY

WORCESTER, MASSACHUSETTS 01610

USA

E-mail: gmaschler@clarku.edu
DEPARTMENT OF MATHEMATICS

UNION COLLEGE

SCHENECTADY, NEW YORK 12308

USA

E-mail: tonnesec@union.edu 